Physical attractiveness biases judgments pertaining to the moral domain of purity

Christoph Klebl, Joshua J. Rhee, Katharine H. Greenaway, Yin Luo, Brock Bastian University of Melbourne

Corresponding author: Christoph Klebl, Melbourne School of Psychological Sciences, University of Melbourne, Redmond Barry Building, Parkville Campus, 3010 Melbourne, Australia, cklebl@student.unimelb.edu.au.

Declaration of interest: none.

This research has been approved by the Human Ethics Research Committee (HREC 1955975.1) at the University of Melbourne.

MANUSCRIPT IS CURRENTLY UNDER REVISION 


\begin{abstract}
Research on the Beauty-is-Good stereotype shows that unattractive people are perceived to have worse moral character than attractive individuals. Yet research has not explored what kinds of moral character judgments are particularly biased by attractiveness. In this work, we tested whether attractiveness particularly biases moral character judgments pertaining to the moral domain of purity, beyond a more general halo effect. Across four pre-registered studies $(N=1,778)$, we found that unattractive (vs. attractive) individuals were judged to be more likely to engage in purity violations compared to harm violations and that this was not due to differences in perceived moral wrongness, weirdness, or sociality between purity and harm violations. The findings shed light on how physical attractiveness influences moral character attributions, suggesting that physical attractiveness particularly biases character judgments pertaining to the moral domain of purity.
\end{abstract}

Keywords: attractiveness, Beauty-is-Good stereotype, moral character, purity, disease avoidance 


\section{Physical attractiveness biases judgments pertaining to the moral domain of purity}

When engaging in social interactions, people are motivated to quickly assess others' moral character in order to determine whether the person is an ally or threat (Brambilla et al., 2021; Goodwin et al., 2014). While people often make accurate judgments of another's moral character traits after only short exposure to their behavior (Ambady \& Rosenthal, 1992; Carney et al., 2007), these quick judgments are also apt to be misled by character-irrelevant information. Most notably, physical attractiveness has been found to positively bias the attribution of desirable character traits such as moral traits (Dion et al., 1972; Klebl et al., in press; Wilson \& Eckel, 2006). Yet there has been no work examining whether-beyond this more general halo effect - there are particular domains of moral character judgment that are particularly biased by attractiveness. Based on evidence showing unattractiveness elicits the emotion of disgust (Klebl, Greenaway, et al., 2021; Park et al., 2003; Park et al., 2013; Ryan et al., 2012), and attractiveness evokes perceptions of purity (Klebl, Luo, et al., 2021), we propose that physical attractiveness may particularly bias moral character judgments pertaining to the purity domain (Graham et al., 2009). Specifically, we suggest that people may judge unattractive (vs. attractive) individuals to be more likely to engage in moral purity violations (e.g., spitting on the ground in public) than in non-purity violations (e.g., pinching someone's arm; Inbar \& Pizarro, 2014).

\section{Moral Character and the Beauty-is-Good Stereotype}

Forming fast and intuitive global impressions of others allows us to quickly generate expectations about how they will act, and in turn inform our emotional and behavioral reactions towards them (Fiske et al., 2002). Growing evidence suggests that, when forming such impressions, individuals may place primacy on information pertaining to the target's moral character (for review see: Brambilla et al., 2021). For example, individuals tend to use 
information on moral traits over sociability or competence when forming a global impression of another person (Brambilla et al., 2012).

Research on the Beauty-is-Good stereotype, however, has revealed that moral character evaluations can be biased by the target's physical appearance (Dion et al., 1972). Whereas early meta-analyses found no evidence of physical attractiveness biasing judgments of 'character' and 'integrity' (Eagly et al., 1991; Feingold, 1992), subsequent empirical studies have found consistent evidence that attractiveness biases moral character attributions (Klebl et al., in press; Wheeler and Kim, 1997; Wilson \& Eckel, 2006; Zebrowitz \& Franklin, 2014). For example, people attribute prototypical moral traits (e.g., fairness or concern for others) more strongly to attractive than to unattractive individuals (Klebl et al., in press). Furthermore, attractive individuals are perceived as more trustworthy and earn more in the first stage of a trust game than unattractive individuals (Wilson \& Eckel, 2006). Even children as young as 8 years of age perceive attractive faces as more trustworthy than unattractive faces (Ma, Xu, et al., 2015), and children trust information provided by an attractive person to a greater extent than information provided by an unattractive person (Bascandziev \& Harris, 2014). The notion that physical attractiveness influences moral character attributions is consistent with neuroimaging studies that have found common neural regions implicated in facial attractiveness judgments and judgments of moral acts (Heinzelmann et al., 2020; Tsukiura \& Cabeza, 2010). Finally, there is a rich tradition of historical evidence and philosophical theorizing that argues beauty and goodness are closely linked with each other (see Henderson, 2015). For example, Plotinus proposed that virtue leads to the ultimate form of beauty by purifying the soul (Gerson, 2017) and Kant claimed that beauty is the 'symbol of morality' (Kant, 1790/2000, p. 225). 


\section{Physical Attractiveness and the Moral Domain of Purity}

The Beauty-is-Good stereotype is typically understood as a halo effect— - that is, a positive evaluation (i.e., attractiveness) that influences unrelated evaluations (e.g., moral character) in a positive direction (Nisbett \& Wilson, 1977; Thorndike, 1920). However, physical attractiveness does not bias all trait attributions uniformly. For example, metaanalyses have revealed large effect sizes for attributions of social competence and sexual warmth, and small to medium effect sizes found for judgments of intelligence and mental health (Eagly et al., 1991; Feingold, 1992). Moreover, traits that are part of the same cognitive associative network are more strongly impacted by physical attractiveness (Forgas \& Laham, 2016). For example, people associate attractiveness with traits that co-occur in daily life (e.g., popularity). Finally, recent evidence has found that attractiveness biases moral character judgments more strongly than attributions of non-moral traits (Klebl et al., in press). These authors suggest that because people are especially sensitive to cues regarding moral traits, they over-rely on heuristic cues when judging moral character in first impressions.

A tacit assumption in previous work is that physical attractiveness will positively influence moral traits in all domains equally. In the present investigation we extend this work by examining whether there are particular moral domains in which beauty exerts an especially strong influence on information processing. Person perception judgments in the moral domain are made on a number of dimensions, notably judgments of harm (e.g., the degree to which people harm others) and purity (e.g., the degree to which people associate with disgusting objects or behaviors; Graham et al., 2009). Despite the importance of distinguishing between these two domains of moral character judgments, this level of specificity has not been present in studies investigating the degree to which physical attractiveness biases such judgments. We seek to address this gap in understanding, and make 
the case in four studies that this effect is likely to be stronger for judgments of purity than harm.

The purity domain is distinct from other moral domains such as harm intuitions which are implicated in people's condemnation of harm inflicted upon sentient beings (Graham et al., 2009; however, see Gray et al., 2012). There is a significant body of work showing that in order to minimize the risk of being exposed to pathogens - that is, microorganisms such as viruses that can cause disease (Wolfe et al., 2007)—people moralize acts that are associated with disease threat, such spitting on a sidewalk or not washing one's hands after using the bathroom (Horberg et al., 2009; Inbar \& Pizarro, 2014; Rozin, 1999; Tybur et al., 2009). People often morally condemn others who commit purity violations even if the act does not directly cause harm to another person (Haidt et al., 1993). For example, people condemn consumption of prohibited food (e.g., a steak made of human muscle cells; Gutierrez \& Giner-Sorolla, 2007; Inbar \& Pizarro, 2014).

There is indirect evidence that physical attractiveness may particularly bias judgments of acts associated with pathogen threat. A growing body of literature suggests that physical attractiveness is linked to the degree to which individuals are perceived to present a pathogen threat (Klebl, Greenaway, et al., 2021; Klebl, Luo, et al., 2021; Kurzban \& Leary, 2001; Park et al., 2003; Ryan et al., 2012; Tapp et al., 2020). While physical attractiveness is not associated with disease in most instances, the disease-avoidance system is over-inclusive and thus also responds to cues that are merely correlated with the presence of disease (Kurzban \& Leary, 2001; Park et al., 2003). This over-inclusiveness ensures that the disease-avoidance system is biased toward making less costly false-positive errors (i.e., judging a healthy person to be infectious), while avoiding false-negative errors (i.e., judging an infectious person to be healthy) that may prove fatal (Kurzban \& Leary, 2001). For example, Klebl, Greenaway, et al. (2021) found that people experience more disgust towards unattractive individuals than 
attractive individuals. Crucially, this appears not to be simply due to shared negative valence between unattractiveness and disgust, as unattractive faces have been found to elicit disgust — but not fear or sadness — when controlling for generalized negativity (Klebl, Greenaway, et al., 2021). Moreover, facial attractiveness has been linked to perceptions of healthiness (Rhodes, 2006) and found to evoke perceptions of purity ("This face makes me think of something pure"; Klebl, Luo, et al., 2021).

\section{The Present Studies}

In the present studies, we investigate whether-beyond the general effect of physical attractiveness on judgments of moral character-attractiveness may especially influence character judgments in specific domains. We hypothesized that attractiveness particularly biases judgments pertaining to the moral domain of purity compared to moral judgments unrelated to purity. This possibility is supported by evidence showing that unattractiveness elicits the emotion of disgust (Klebl, Greenaway, et al., 2021; Park et al., 2013; Ryan et al., 2012) and attractiveness evokes perceptions of purity (Klebl, Luo, et al., 2021). Furthermore, the present studies extend previous work by looking specifically at whether physical attractiveness biases the degree to which people expect others to engage in moral norm violating behaviors, beyond the more general trait attributions assessed in past work (Klebl et al., in press).

In order to test this hypothesis, we investigated in four pre-registered studies whether unattractive (vs. attractive) individuals are perceived as more likely to engage in purity violations compared to harm violations. In Study 1a, we examined this in a paired-evaluation design in which participants were presented with pairs of unattractive and attractive faces and judged which of the depicted people would be more likely to engage in various impure acts or harmful acts. In Study 1b, we tested whether the effect was driven by the lower half of the attractiveness spectrum by contrasting unattractive and average-looking faces. In Study 2, we 
used a more ecologically valid separate-evaluation design to compare attractive, averagelooking, and unattractive individuals. To rule out the possibility the effect was due to valence, we matched violations on perceived wrongness.

Finally, in Study 3, we aimed to reinforce confidence in our theorizing that the observed effect can be explained by the link between physical attractiveness and perceptions of purity and impurity. Therefore, we replicated Study 2, while matching purity and harm violations on perceived weirdness (i.e., the degree to which the act is atypical or unusual), sociality (i.e., the degree to which the act involves other people), and moral wrongness.

\section{Study 1a}

In Study 1a, we hypothesized that unattractive individuals would be judged as more likely to engage in moral violations overall compared to attractive individuals. Furthermore, we hypothesized that unattractive (vs. attractive) individuals would be judged as more likely to engage in impure acts compared to harmful acts. We tested this hypothesis by presenting participants with pairs of faces (one unattractive and one attractive) and asking them to judge which of the depicted people would be more likely to engage in a purity violation or harm violation, respectively.

\section{Method}

Participants. An a priori power analysis revealed that a minimum sample of $N=264$ was required to detect a small effect size at .80 power $(\alpha=.05$; Faul et al., 2009). We recruited 300 participants living in the USA from MTurk. Three hundred and three participants $\left(M_{\mathrm{age}}=36.5, S D=10.4\right.$, range $=20-74 ; 69.3 \%$ White, $21.8 \%$ Black, $4.3 \%$ Asian, 3.3\% Native Hawaiian or Pacific Islander, 1.3\% Native American; 71.0\% college degree, $19.8 \%$ professional degree, $7.6 \%$ high school graduate, $1.3 \%$ doctorate, $0.3 \%$ less than high school $)^{1}$ completed the survey and were compensated US $\$ 0.40$ for a 2 -minute study. The

\footnotetext{
${ }^{1}$ Data on participants' gender was inadvertently not collected in Studies 1a and 1b.
} 
study was pre-registered (available at https://osf.io/qystm/?view_only=aa956910572840ffa392990d30b4f163). The data and materials of all studies are available at https://osf.io/m5g72/?view_only=d738c44e5de6487fa9d45c0ed07be5c8.

Materials and Procedure. Participants were randomly presented with nine pairs of human faces (two White female, two White male, two Asian female, one Asian male, one Black female, and one Black male) taken from Ma, Correll, et al. (2015), with each pair containing one attractive and one unattractive variant that was matched on sex and ethnicity. ${ }^{2}$ We selected the most attractive and unattractive faces based on the norming data provided by Ma, Correll, et al. (2015). However, we excluded faces that had cues of disease such as sweatiness. The position of the faces within each pair was randomized. The face on the left was labelled "Person A" and the face on the right was labelled "Person B".

Each pair of faces was presented twice: once together with a harm violation vignette and once together with a purity violation vignette. In total, we used nine harm violation and nine purity violation vignettes (see Table 1) taken from Chakroff and Young (2014). A paired-evaluation method was employed, such that participants were required to indicate on a slider $(-100=$ more likely Person A, $0=$ equally likely, $100=$ more likely Person $B)$ who of the depicted individuals would be more likely to engage in the respective act (e.g., Who of the depicted persons would be more likely to kick someone in the shin).

\footnotetext{
${ }^{2}$ For all studies, images of faces are displayed in the supplemental materials.
} 


\section{Table 1}

Harmful and impure acts used in Studies $1 \mathrm{a}$ and $1 \mathrm{~b}$. The vignettes were taken from Chakroff \& Young (2014).

\begin{tabular}{ll}
\hline Harmful acts & Impure acts \\
\hline Kick someone in the shin & Lick someone's shoe \\
Scratch someone on the arm & Pour urine on oneself \\
Prick someone's hand with a needle & Pick up dog poop barehanded \\
Poke someone in the eye & Step in vomit barefoot \\
Burn someone on the arm & Taste earwax \\
Hit someone's finger with a hammer & Drink cow blood \\
Cut someone's cheek with a razor & Pick up a snot-filled Kleenex \\
Pinch someone hard on the arm & Pick up a used Band-Aid \\
Whip someone with a belt & Eat a worm
\end{tabular}

\section{Results}

Thirty-three participants were excluded from the analyses. ${ }^{3}$ Thirty participants failed the attention check and three did not consent to their data being used. Linear mixed-effects models with participants and stimuli as random intercepts were used in all analyses $(\mathrm{R}$ package lme4; Bates et al., 2014). In order to test whether unattractive individuals were judged to be more likely to engage in violations overall, we tested whether ratings deviate from zero. Positive values indicate a higher judged likelihood that unattractive individuals would engage in the violations. As expected, unattractive individuals were judged to be more likely to engage in immoral acts compared to attractive individuals $(M=21.0, S D=28.4), b$

\footnotetext{
${ }^{3}$ Data exclusions were preregistered in all studies. Excluding participants had no substantive impact on the results (see supplemental materials).
} 
$=20.95, S E=2.29, t(27.37)=9.13, p<.001,95 \%$ CI $[16.37,25.53]$. Furthermore, to test whether there were differences between the judged likelihood to engage in impure and harmful acts, we included violation type $(0=$ harmful, $1=$ impure $)$ into the model. As hypothesized, the difference in the rated likelihood to engage in moral violations between unattractive and attractive individuals was significantly greater for impure acts $(M=27.5, S D$ $=33.0)$ than for harmful acts $(M=14.5, S D=29.8), R^{2}=.01, b=13.01, S E=1.44, t(4581)=$ $9.04, p<.001,95 \%$ CI $[10.19,15.83]$ (see Figure 1). ${ }^{4}$

In summary, we found that unattractive individuals were judged as more likely to engage in moral violations than attractive individuals, consistent with a physical attractiveness halo effect. Furthermore, we found that unattractive (vs. attractive) individuals were judged to be more likely to engage in impure acts compared to harmful acts. This suggests that beyond the general halo effect, there is a specific domain in which attractiveness more strongly influences judgments of moral character: Physical attractiveness is linked to the disease-avoidance system (Klebl, Greenaway, et al., 2021; Klebl, Luo, et al., 2021; Ryan et al., 2012), and as a result, people expect unattractive individuals to be more likely to engage in violations that are impure, relative to attractive individuals who are perceived as not presenting a contamination threat.

\footnotetext{
${ }^{4}$ For all studies, we exploratively tested whether the effects are moderated by the faces' and participants' gender (see supplemental materials).
} 


\section{Figure 1}

Linear mixed-effects model with violation type (harmful vs. impure) as independent variable and the likelihood to engage in violations as the dependent variable (Study $1 a ; N=270$ ).

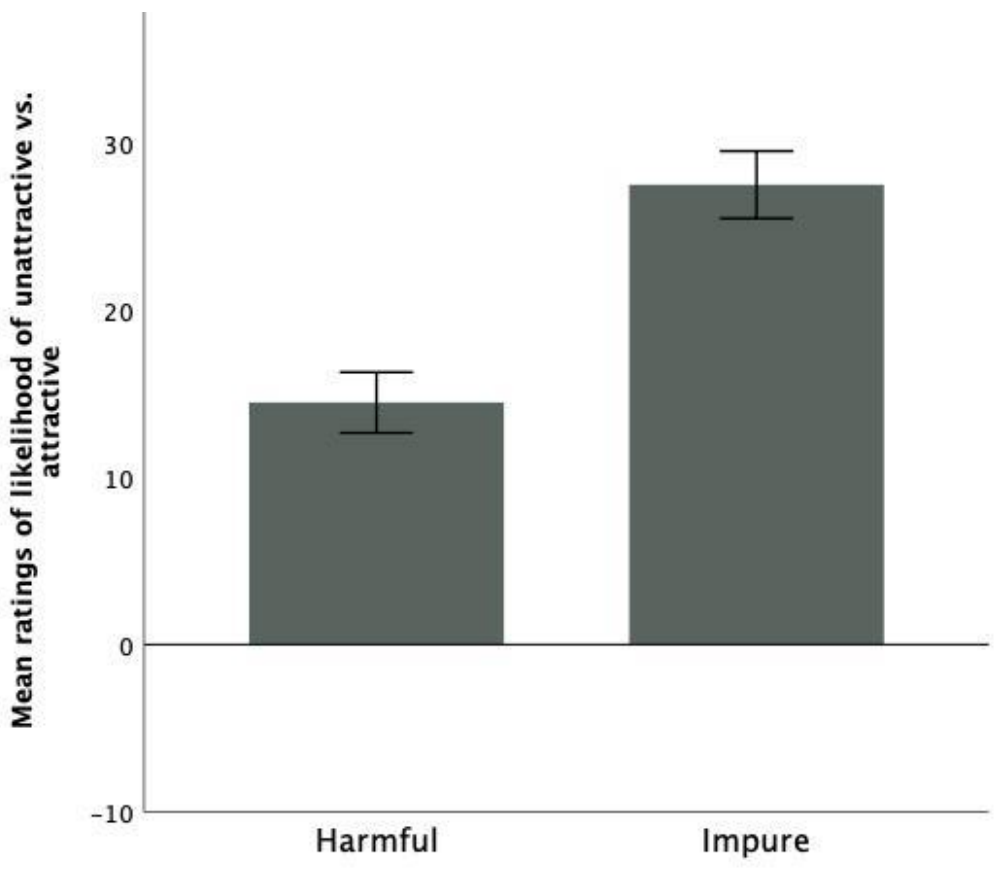

Note. Positive values indicate a higher likelihood for unattractive individuals to engage in violations compared to attractive individuals. Error bars represent standard errors.

\section{Study 1b}

In Study 1a, we found that people judge unattractive compared to attractive individuals to be more likely to engage in impure acts than in harmful acts. However, the directionality of this effect remains unclear. Based on previous research suggesting that unattractiveness is more disadvantageous than attractiveness is advantageous (Griffin \& Langlois, 2006), and that the negative effect of skin blemishes on trustworthiness judgments is greater than the positive influence of skin smoothness on trustworthiness judgments (Jaeger et al., 2018), our secondary hypothesis was that unattractiveness would drive the observed effect. Therefore, in Study 1b, we investigated whether unattractive individuals would be judged to be more likely to engage in impure (vs. harmful) acts compared to average-looking individuals. 


\section{Method}

Participants. As in Study 1a, we recruited 300 participants living in the USA from MTurk, and 276 participants $\left(M_{\text {age }}=35.7, S D=9.3\right.$, range $=22-70 ; 59.4 \%$ White, $35.1 \%$ Black, 2.9\% Asian, 0.7\% Native American, 0.4\% Native Hawaiian or Pacific Islander; 65.5\% college degree, $27.6 \%$ professional degree, $5.1 \%$ high school graduate, $1.5 \%$ doctorate, $0.4 \%$ less than high school) completed the 2-minute survey in return for US\$0.50. The preregistration can be found at https://osf.io/2sw86?view_only=76e7e150297e46e6a2456a0b9181aedc.

Materials and Procedure. We employed the same design and procedure as in Study 1a but presented participants with nine pairs of either unattractive or average-looking faces (Ma, Correll, et al., 2015). The unattractive faces were identical to those used in Study 1a. We selected average-looking faces by splitting the dataset in half along their attractiveness ratings for each sex and ethnicity and selecting the faces nearest to the midpoints. We used the same vignettes as in Study 1a (see Table 1).

\section{Results}

We excluded 39 participants from the analyses. ${ }^{5}$ Thirty-one participants failed the attention check and eight participants did not consent to their data being used. We used linear mixed-effects models in all analyses with participants as random intercept in the first analysis and participants and stimuli as random intercepts in the second analysis (i.e., the maximal random effect structure justified by the data).

As expected, unattractive individuals were judged to be more likely to engage in immoral acts compared to average-looking individuals $(M=14.3, S D=29.9), b=14.31, S E$ $=2.09, t(70.08)=6.84, p<.001,95 \%$ CI $[10.18,18.44]$. However, contrary to our secondary

\footnotetext{
${ }^{5}$ Excluding participants had no substantive impact on the results (see supplemental materials).
} 
hypothesis, the difference between unattractive and average-looking individuals in judged likelihood to engage in moral violations did not significantly differ between impure acts $(M=$ $14.5, S D=32.7)$ and harmful acts $(M=14.1, S D=33.3), R^{2}<.01, b=0.43, S E=1.67$, $t(4028)=0.26, p=.789,95 \% \mathrm{CI}[-3.69,2.83]$.

In summary, we did not find that unattractive individuals were judged to be more likely to engage in impure (vs. harmful) acts compared to average-looking individuals. This suggests that unattractive individuals were not judged differently to average-looking people. Rather, it could be that people judge attractive individuals to be less likely to engage in impure (vs. harmful) compared to average-looking individuals and unattractive individuals. We explored this possibility in Study 2.

\section{Study 2}

In the previous two studies, we used paired evaluations of faces, such that people evaluated unattractive and attractive faces against one another. However, people evaluate stimuli differently in joint and separate evaluations (Hsee et al., 1999) and joint comparisons arguably have a lower ecological validity. Therefore, in Study 2, we aimed to replicate our previous findings using separate evaluations of faces that were not presented in relation to one another. We also matched the vignettes in perceived wrongness in order to rule out the possibility that the observed effect can be explained by differences in valence between impure and harmful acts. Moreover, we employed a full comparison of attractive, averagelooking and unattractive faces.

\section{Method}

Participants. We determined $a$ priori that a sample size of $N=395$ would allow for an $80 \%$ chance of detecting a small effect size at $\alpha=.05$ (Faul et al., 2009). In order to secure sufficient power, we recruited 600 participants ( 231 female, 368 male, 1 non-binary; $M_{\text {age }}=$ $35.7, S D=10.2$, range $=22-71 ; 72.2 \%$ White, $22.2 \%$ Black, $2.1 \%$ Native Hawaiian or Pacific 
Islander, 2.0\% Asian, 1.3\% Native American; 64.0\% college degree, 24.1\% professional degree, $10.4 \%$ high school graduate, $0.8 \%$ doctorate, $0.7 \%$ less than high school) living in the USA via MTurk in return for US\$0.60 for completing a 3-minute study. The study was preregistered (available at https://osf.io/8xygz?view_only=1cc6a6c2dd184c02a9a5a23490403998).

Materials and Procedure. We employed a mixed design, with attractiveness (attractive vs. average-looking vs. unattractive) as a between-subject variable, and violation type (impure vs. harmful) as a within-subject variable. Participants were randomly assigned to one of the three attractiveness conditions and presented with six faces in a randomized order. As in the previous studies, the stimuli were selected from the Chicago Face Database (Ma, Correll, et al., 2015; see Figure 2 and supplemental materials) and were matched in ethnicity (Asian, Black, and White) and gender (male and female). All faces were presented one at a time and each face was presented four times: twice in combination with a harm violation and twice in combination with a purity violation. The assignment and order of presentation was randomized. Participants were asked to indicate on a slider $(-100=m u c h$ less likely, $0=$ equally likely, $100=$ much more likely) how likely the depicted person would engage in the act described in the respective vignette, compared to an average person.

\section{Figure 2}

Sample images used in Study 2 (Ma, Correll, et al., 2015). Displayed from left to right: attractive face, average-looking face, and unattractive face.
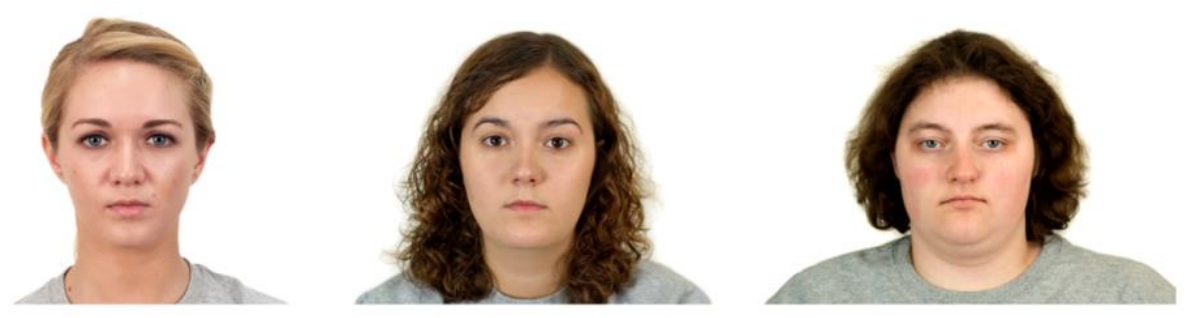
To match the vignettes on perceived wrongness, we tested in a pilot study $(N=50)$ the 18 vignettes from Chakroff \& Young (2015) and 40 vignettes written by research assistants. We selected 12 harm and 12 purity violations with matched mean perceived wrongness ratings (both 3.55 on a 6-point scale; see Table 2). Harm violations were judged as more harmful than purity violations, and purity violation were judged as more impure than harm violations ( $p$ s $<.001$; see supplemental materials).

\section{Table 2}

Harmful and impure acts used in Study 2.

\begin{tabular}{ll}
\hline Harmful acts & Impure acts \\
\hline Grabbing someone's arm very hard & Tasting earwax \\
Pulling someone's hair & Sniffing their armpits indiscreetly \\
Calling someone incompetent & Spitting on the street \\
Pinching someone's arm & Picking up dog poop barehanded \\
Joking about an embarrassing incident that & Scratching their feet and then picking up food \\
happened to someone else & \\
Laughing about someone else's clothes & Clipping their nails in the tram \\
Laughing at someone else's taste in music & Spitting on the floor at home \\
Swearing at strangers & Eating food with mold on it \\
Stepping on someone's foot deliberately & Wearing someone else's unwashed underpants \\
Laughing at someone who fell on the floor & Picking dead skin off their own feet in public \\
Whipping someone with a belt & Pouring urine on themselves \\
Shoving someone out of the way & Not wiping their bottom after using a toilet \\
\hline
\end{tabular}




\section{Results}

We excluded 112 participants from the analyses. ${ }^{6}$ Ninety-six participants failed the attention check and fifteen participants did not consent to their data being used. In all studies, linear mixed-effects models with participants and stimuli as random effects were used.

We first ran a linear mixed-effects model with attractiveness $(0=$ attractive, $1=$ average-looking, $2=$ unattractive $)$ and violation type $(0=$ harmful, $1=$ impure $)$ as predictors and judged likelihood to engage in acts as the dependent variable (see Figure 3). The model explained a variance of $R^{2}=.63$. There was no significant main effect of attractiveness, $b=$ $3.27, S E=2.02, t(539.53)=1.62, p=.106,95 \% \mathrm{CI}[-0.69,7.22]$. However, there was a significant main effect of violation type, such that people were judged to be more likely to engage in harmful acts than in impure acts, $b=-28.97, S E=1.18, t(5361)=-24.61, p<.001$, 95\% CI [-31.28, -26.66]. As hypothesized, we found an interaction effect between attractiveness and violation type, $b=5.95, S E=0.91, t(5361)=6.53, p<.001,95 \% \mathrm{CI}[4.16$, 7.73].

We then conducted simple slope analyses testing at each level of violation type whether attractiveness predicted perceived likelihood to engage in violations (for means and standard deviations see Table 3). There was no significant effect of attractiveness on perceived likelihood to engage in harmful acts, $R^{2}<.01, b=3.27, S E=1.85, t(486)=1.77, p$ $=.078,95 \% \mathrm{CI}[-0.25,6.89]$. However, there was a significant effect of attractiveness on perceived likelihood to engage in impure acts, $R^{2}=.02, b=9.22, S E=2.33, t(486)=3.96, p$ $<.001,95 \% \mathrm{CI}[4.65,13.78]$. Tukey-adjusted post-hoc tests revealed that attractive individuals were judged to be less likely to engage in impure acts compared to unattractive individuals, $p<.001$, as well as compared to average-looking individuals, $p=.024$. However,

\footnotetext{
${ }^{6}$ As in the previous studies, excluding participants had no substantive impact on the results (see supplemental materials).
} 
there was no significant difference between average-looking faces and unattractive faces, $p=$ .390. The significant interaction effect shows that attractive individuals (compared to unattractive and average-looking individuals) were judged to be less likely to engage in impure acts than in harmful acts.

In summary, we found that attractive (vs. average-looking and unattractive) individuals were judged to be less likely to engage in impure acts compared to harmful acts that were matched in perceived wrongness. This suggests that attractiveness particularly biases moral character judgments pertaining to the moral domain of purity, relative to judgments of non-purity violations. Contrary to our secondary hypothesis, but consistent with Study $1 b$, the present study suggests that the observed effect is due to attractive individuals being judged to be less likely to engage in impure (vs. harmful) acts compared to averagelooking individuals, whereas average-looking and unattractive individuals were judged similarly to each other.

\section{Table 3}

Means and standard deviations of ratings of harmful and impure acts for attractive $(N=$ 164), average-looking $(N=161)$, and unattractive $(N=163)$ faces $($ Study 2$)$.

\begin{tabular}{lcccccc}
\hline & \multicolumn{2}{c}{ Attractive } & \multicolumn{2}{c}{ Average } & \multicolumn{2}{c}{ Unattractive } \\
& $M$ & $S D$ & $M$ & $S D$ & $M$ & $S D$ \\
\hline Harmful & 12.5 & 31.9 & 16.0 & 35.6 & 19.1 & 32.6 \\
Impure & -17.4 & 41.3 & -5.1 & 43.9 & 1.0 & 41.1 \\
& & & & & & \\
\hline
\end{tabular}




\section{Figure 3}

Linear mixed-effects model with attractiveness (attractive vs. average-looking vs.

unattractive) and violation type (harmful vs. impure) as independent variables; and the likelihood to engage in violations as the dependent variable (Study 2).

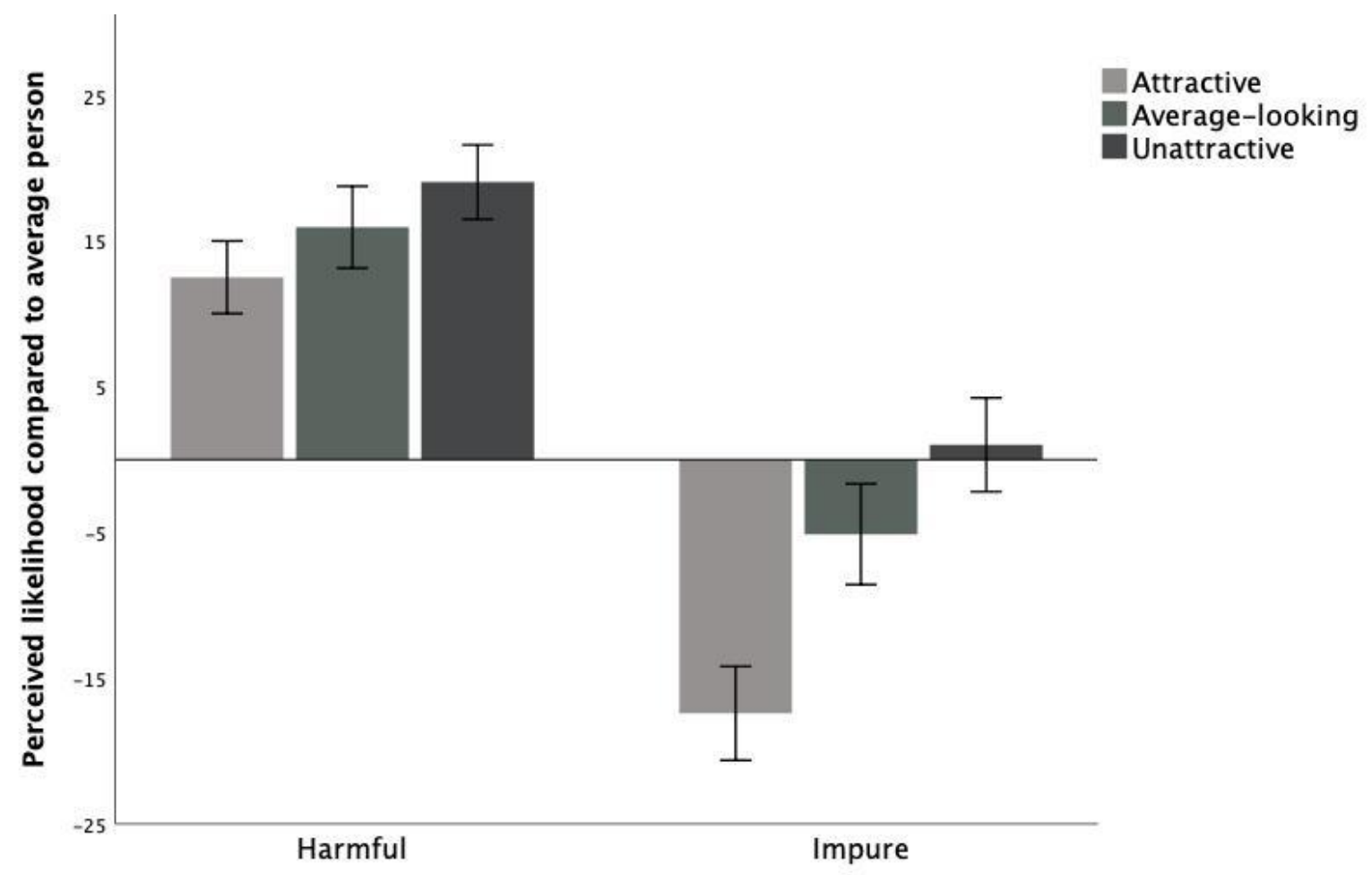

Note. Error bars represent standard errors.

\section{Study 3}

Study 2 replicated the finding that physical attractiveness (vs. unattractiveness) particularly biases character judgments pertaining to the moral domain of purity and this was not due to differences in moral wrongness between purity and harm violations. However, previous work suggests that purity and harm violations also differ in terms of their weirdness (i.e., impure acts are perceived as more abnormal than harmful acts; Gray \& Keeney, 2015) and their sociality (i.e., harmful acts more likely involve other people; Chakroff et al., 2013). Therefore, in Study 3, we used harm and purity violations that were matched with respect to their perceived moral wrongness, weirdness, and sociality. 
Furthermore, not all purity violations are directly associated with pathogen threat. It has been suggested that the sub-category of sexual purity has evolved to serve the specific function of avoiding sexual contacts with individuals of low mate quality (Tybur et al., 2009). For example, people typically condemn non-normative sexual practices such as incest or promiscuity (Gutierrez \& Giner-Sorolla, 2007; Tybur et al., 2009). Therefore, we explored whether judgments of sexually impure acts would present a boundary condition to the observed effect. Based on our theorizing that attractiveness particularly biases judgments pertaining to the moral domain of purity due to the unique link between attractiveness and the disease-avoidance system (Klebl, Greenaway, et al., 2021; Klebl, Luo, et al., 2021; Park et al., 2013), we hypothesized that sexually impure acts would be judged similarly to harm violations. Finally, to provide further confidence in our theorizing, we tested whether physical attractiveness predicts perceptions of purity and feelings of disgust.

\section{Method}

Participants. As in Study 2, we recruited 600 participants to a have sufficient power to detect a small effect size at $\alpha=.05$. Participants were recruited from Prolific and lived in the USA or UK. The final sample comprised 599 participants (394 female, 199 male, 5 nonbinary, 1 missing data; $M_{\mathrm{age}}=34.5, S D=12.3$, range $=18-70 ; 85.1 \%$ White, $7.8 \%$ Asian, $2.2 \%$ Black, $4.8 \%$ other ethnicities; $64.3 \%$ college degree, $17.4 \%$ professional degree, $15.0 \%$ high school graduate, $2.0 \%$ doctorate, $1.3 \%$ less than high school). The pre-registration is available at https://osf.io/3gbfs/?view_only=7792b32adbb945b89c1e53696d95e37a.

Materials and Procedure. In a pilot study $(N=399)$, we tested 91 vignettes and selected five harm violations, five purity violations and three sexual purity violations that were matched in moral wrongness ("The act is morally wrong."), weirdness ("How atypical is this act? That is, it is weird, strange and/or unusual."'), and sociality ("The act involves 
another person"; see Table 4 for the selected violations; see supplemental materials for the pilot study).

In the main study, participants were randomly presented with either five attractive, average-looking, or unattractive faces (one White female, one White male, one Asian female, one Asian male, and one Black male face; Ma, Correll, et al., 2015). Each face was presented once with a harm violation and once with a purity violation. Three of the faces (White female, White male, and Asian female) were presented with sexual purity violations. As in Study 2, the assignment of faces and violations as well as the order of presentation was randomized, and participants were asked to indicate on a slider $(-100=$ much less likely, $0=$ equally likely, $100=$ much more likely) how likely the person would engage in the act compared to an average person.

As a manipulation check, participants were then presented with the violations only and asked to indicate on a six-point scale $(1=$ Not at all, $6=$ Very much so $)$ the degree to which they find the respective act harmful ("The act is harmful. That is, it involves physical and/or emotional suffering.") and impure ("The act is impure. That is, it involves sinfulness, indecency and/or dirtiness"; Gray \& Keeney, 2015). Finally, participants were presented with the faces only and asked to indicate each face's attractiveness relative to other people of the same race and gender on a scale from 0 (Extremely attractive) to 10 (Extremely unattractive). Furthermore, they were asked to indicate on a 11-point scale $(0=$ Not at all, $10=$ Very much so) the degree to which the face makes them think of something pure ("This face makes me think of something pure.") and makes them feel disgusted ("This face makes me feel disgusted.").

\section{Results}

We excluded two participants because they failed the attention check and six participants because they did not consent to their data being used. 
Manipulation checks. We used paired-samples $t$-tests to test whether participants judged harm violations as more harmful and less impure than purity and sexual purity violations. As expected, harmful acts $(M=4.8, S D=0.8)$ were judged as significantly more harmful than impure acts $(M=3.3, S D=1.1), t(590)=34.45, p<.001,95 \%$ CI $[1.41,1.58]$, and sexually impure acts $(M=3.3, S D=1.2), t(590)=27.96, p<.001,95 \%$ CI $[1.40,1.61]$. Impure acts $(M=4.6, S D=1.0)$ were judged as significantly more impure than harmful acts $(M=3.6, S D=1.2), t(588)=21.71, p<.001,95 \%$ CI $[0.92,1.10]$, and sexually impure acts $(M=4.6, S D=1.1)$ were judged as more impure than harmful acts, $t(589)=16.89, p<.001$, 95\% CI $[0.87,1.10]$. However, there was no significant difference between impure and sexually impure acts in either perceived harmfulness, $t(590)=0.28, p=.783,95 \% \mathrm{CI}=[-$ $0.74,0.10]$, or impurity, $t(589)=0.67, p=.504,95 \% \mathrm{CI}[-0.06,0.11]$.

Linear mixed-effects models with stimuli and participants as random intercepts were used to test whether attractive, average-looking, and unattractive faces differed in their perceived attractiveness (for descriptive statistics see Table 5). As expected, attractive faces were judged as more attractive than unattractive faces $b=1.22, S E=0.05, t(588)=22.47, p<$ $.001,95 \% \mathrm{CI}[1.12,1.33]$, and average-looking faces, $b=0.55, S E=0.05, t(588)=10.00, p<$ $.001,95 \%$ CI $[0.44,0.65]$. Average-looking faces were judged as more attractive than unattractive faces, $b=0.68, S E=0.05, t(588)=12.49, p<.001,95 \%$ CI $[0.57,0.78]$.

Disgust and purity judgments. Linear mixed-effects models were used to test whether there were differences in participants' perceptions of purity and experiences of disgust towards faces as a function of the faces' attractiveness (for descriptive statistics see Table 5). Unattractive faces were judged as more disgusting than attractive faces, $b=0.55$, $S E=0.08, t(588)=6.74, p<.001,95 \% \mathrm{CI}[0.39,0.71]$, and average-looking faces, $b=0.48$, $S E=0.08, t(588)=5.89, p<.001,95 \% \mathrm{CI}[0.32,0.64]$. However, there was no significant difference in disgust experiences towards attractive and average-looking faces, $b=0.07, S E=$ 
$0.08, t(588)=0.89, p=.375,95 \% \mathrm{CI}[-0.09,0.23]$. Consistent with this, unattractive faces were judged as less pure than attractive faces, $b=-0.48, S E=0.10, t(588)=-4.90, p<.001$, 95\%CI [-0.67, -0.29], and average-looking faces, $b=-0.47, S E=0.10, t(588)=-4.90, p<$ $.001,95 \% \mathrm{CI}[-0.66,-0.28]$. There was, however, no significant difference in perceptions of purity between average-looking and attractive faces, $b=-0.002, S E=0.10, t(588)=-0.03, p$ $=.977,95 \%$ CI $[-0.20,0.19]$.

Main analyses. We first compared judged likelihood to engage in harmful and impure acts using a linear mixed-effects model with attractiveness $(0=$ attractive, $1=$ average-looking, $2=$ unattractive $)$ and violation type $(0=$ harmful, $1=$ impure $)$ as predictors and judged likelihood to engage in the act as the dependent variable (see Figure 4). The model explained a variance of $R^{2}=.29$. There were no significant main effects of attractiveness, $b=0.70, S E=0.63, t(956.94)=1.11, p=.269,95 \% \mathrm{CI}[-0.54,1.94]$, and violation type, $b=-3.41, S E=4.42, t(8.30)=-0.77, p=.462,95 \%$ CI $[-11.97,5.16]$. As hypothesized, a significant interaction effect between attractiveness and violation type was revealed, $b=3.72, S E=0.59, t(5309)=6.28, p<.001,95 \% \mathrm{CI}[2.56,4.88]$.

We conducted simple slope analyses testing at each level of violation type whether attractiveness predicted judged likelihood to engage in violations (for descriptive statistics see Table 5). No significant effect of attractiveness on likelihood to engage in harmful acts was found, $R^{2}<.01, b=0.70, S E=0.63, t(589)=1.12, p=.264,95 \%$ CI $[-0.53,1.93]$. However, there was a significant effect of attractiveness on likelihood to engage in impure acts, $R^{2}=.03, b=4.42, S E=0.62, t(589)=7.08, p<.001,95 \%$ CI $[3.20,5.65]$. Tukey adjusted post-hoc tests revealed that unattractive individuals were judged as significantly more likely to engage in impure acts than attractive individuals, $p<.001$, and averagelooking individuals, $p<.001$. There was, however, no significant difference between attractive faces and average-looking faces, $p=.130$. 
We then tested whether there were differences between perceived likelihood to engage in harmful and sexually impure acts. In order to compare judgments of harmful and sexually impure acts, we created a new harmfulness variable that averaged judgments of the likelihood to engage in harmful acts for White female, White male, and Asian female faces. We tested a linear mixed-effects model with attractiveness and violation type $(0=$ harmful, 1 $=$ sexually impure) as predictors and judged likelihood to engage in acts as the dependent variable. The model explained a variance of $R^{2}=.25$. There were no significant main effects of attractiveness, $b=0.16, S E=0.72, t(1299.15)=-0.22, p=.820,95 \% \mathrm{CI}[-1.25,1.58]$, and violation type, $b=-3.55, S E=7.20, t(4.11)=-0.49, p=.647,95 \% \mathrm{CI}[-17.18,10.08]$. As expected, there was also no significant interaction between attractiveness and violation type, $b=0.51, S E=0.85, t(2989)=-0.60, p=.551,95 \%$ CI $[-1.16,2.17]$.

In summary, unattractive (vs. attractive) individuals were judged as more likely to engage in impure acts compared to harmful acts matched on perceived wrongness, weirdness, and sociality. Moreover, as predicted, unattractive (vs. attractive and average-looking) individuals were expected to engage to a similar degree in harmful acts and sexually impure acts. Unattractive faces were also judged as more disgusting and less pure compared to attractive and average-looking faces. These findings provide further evidence for our theorizing that physical attractiveness particularly biases judgments pertaining to the moral domain of purity due to its unique link with the disease-avoidance system (Klebl, Greenaway, et al., 2021). Furthermore, consistent with our secondary prediction, but inconsistent with Studies $1 \mathrm{~b}$ and 2, Study 3 suggests that the observed effect was due to unattractive individuals being judged as more likely to engage in impure (vs. harmful) acts compared to average-looking individuals, whereas average-looking and attractive individuals were judged similarly to each other. 


\section{Table 4}

Harmful, impure, and sexually impure acts used in Study 3.

Harmful acts:

1. Pricking someone's hand with a needle.

2. Throwing rocks at cows that are grazing in the local pasture.

3. Making fun of someone for getting dumped by their girlfriend.

4. Throwing a stapler at their colleague who is snoring during their talk.

5. Scratching someone on the arm.

Impure acts:

1. Baking a cake for their family after using the toilet and not washing their hands.

2. Urinating in the wave pool at a crowded amusement park.

3. Secretly using a stranger's toothbrush in a dorm bathroom.

4. Searching through the trash to find another person's discarded underwear.

5. Farting intentionally in an elevator with four other people.

Sexually impure acts:

1. Having sex in public.

2. Masturbating on a city bus.

3. Listening to two strangers having sex.

\section{Table 5}

Means and standard deviations of violation types and ratings of faces for attractive $(N=$ 193), average-looking $(N=197)$, and unattractive $(N=201)$ faces (Study 3).

\begin{tabular}{llcccccc}
\hline & & \multicolumn{2}{c}{ Attractive } & \multicolumn{2}{c}{ Average } & \multicolumn{2}{c}{ Unattractive } \\
& & $M$ & $S D$ & $M$ & $S D$ & $M$ & $S D$ \\
\hline \multirow{2}{*}{$\begin{array}{l}\text { Likelihood of } \\
\text { engaging in act }\end{array}$} & Harmful acts & -13.5 & 26.9 & -13.4 & 22.9 & -10.7 & 24.6 \\
& Impure acts & -19.4 & 27.1 & -14.5 & 23.9 & -1.7 & 23.1 \\
& Sexual acts & -15.1 & 31.1 & -16.9 & 24.6 & -12.4 & 25.7 \\
& Harmful acts* & -8.4 & 30.7 & -10.1 & 27.4 & -7.7 & 27.5 \\
\multirow{2}{*}{ Ratings of faces } & Attractiveness & 6.0 & 1.1 & 4.9 & 1.1 & 3.6 & 1.0 \\
& Disgust & 2.0 & 1.4 & 2.1 & 1.6 & 3.1 & 1.8 \\
& Purity & 4.4 & 2.0 & 4.4 & 2.1 & 3.4 & 1.7 \\
\hline
\end{tabular}

* This variable only includes ratings of White female, White male, and Asian female faces. 


\section{Figure 4}

Linear mixed-effects model with attractiveness (attractive vs. average-looking vs.

unattractive) and violation type (harmful vs. impure) as independent variables; and the likelihood to engage in violations as the dependent variable (Study 3).

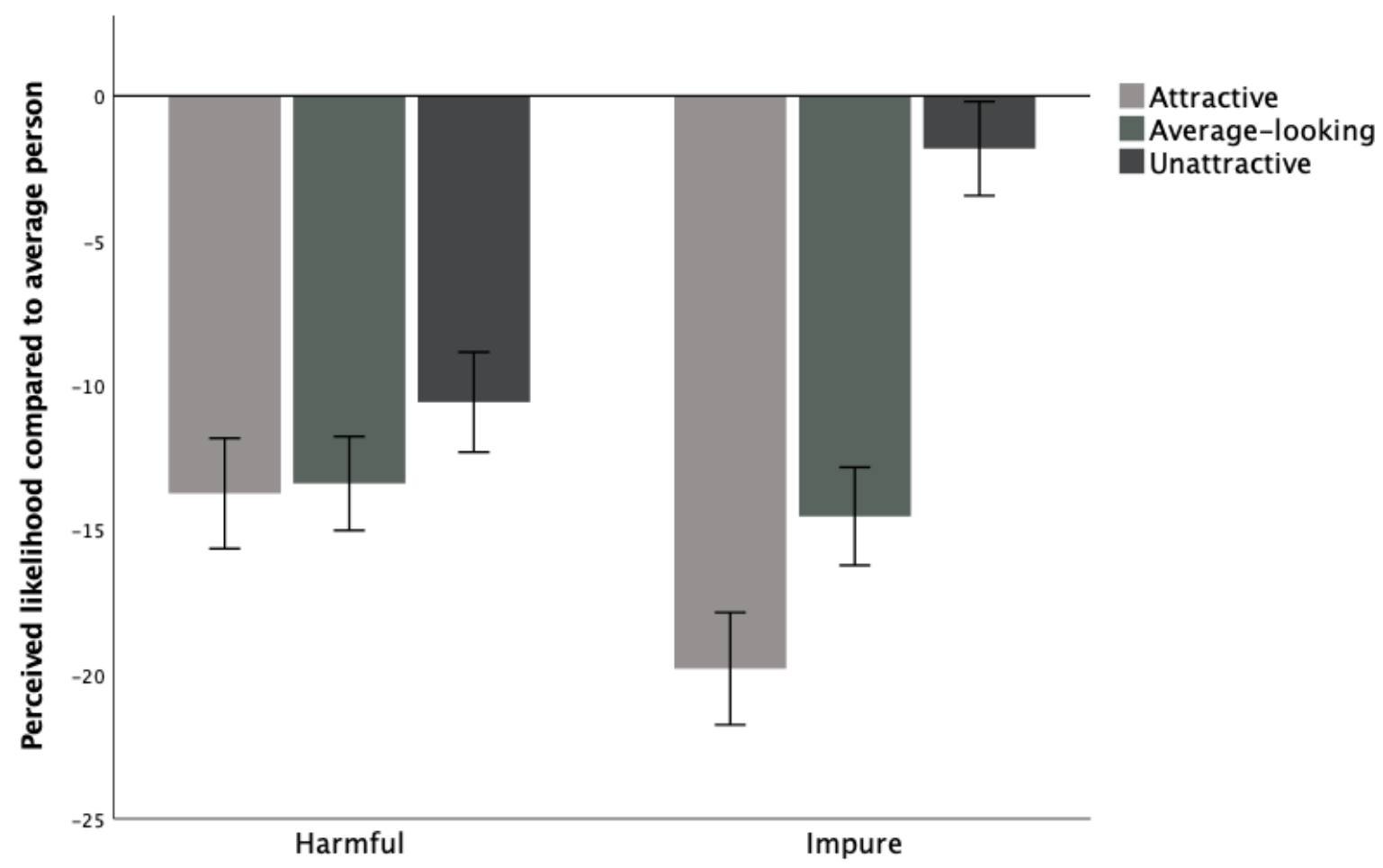

Note. Error bars represent standard errors

\section{General Discussion}

Across four pre-registered studies, we sought to investigate whether physical attractiveness influences moral character judgments in specific domains. We found support for our hypothesis that physical attractiveness particularly biases judgments of purity violations, relative to non-purity violations. In Study 1a, we found in a joint-evaluation design that attractive people were judged to be less likely to engage in moral violations than unattractive individuals, consistent with a physical attractiveness halo effect. Crucially, we demonstrated that unattractive (vs. attractive) individuals were judged to be more likely to engage in impure acts (e.g., eating a worm) compared to harmful acts (e.g., pinching 
someone's arm). Although our main aim was to establish a link between attractiveness and the judged likelihood of engaging in purity violations, we were also interested in whether it was especially unattractiveness or attractiveness that drove this relationship. Contrary to our secondary prediction, in Study 1b, we did not find that unattractive people were judged to be more likely to engage in impure (vs. harmful) acts compared to average-looking individuals. In Study 2, we replicated the effect found in Study 1a employing an ecologically valid separate-evaluation design and matching purity and harm violations in perceived wrongness in order to control for differences in valence between the violation types. Consistent with Study $1 \mathrm{~b}$, attractive individuals were judged as being less likely to engage in impure (vs. harmful) acts compared to average-looking individuals, whereas average-looking and unattractive individuals were judged similarly to each other. Finally, Study 3 replicated that unattractive (vs. attractive) individuals were judged to be more likely to engage in impure acts than in harmful acts while matching the vignettes on perceived wrongness, weirdness, and sociality. Moreover, no differences in judgments of harmful acts and sexually impure acts (i.e., purity violations that are not directly linked to pathogen threat) were found. This provides further support for our theorizing that the observed effect is due to the unique link between physical attractiveness and the disease-avoidance system (Klebl, Greenaway, et al., 2021). Consistent with our secondary prediction, but inconsistent with Studies $1 \mathrm{~b}$ and 2 , unattractive people were judged as more likely to engage in impure (vs. harmful) acts compared to average-looking individuals, whereas average-looking and attractive individuals were judged similarly to each other.

Our findings extend previous research on the Beauty-is-Good stereotype which demonstrates that people attribute moral traits such as trustworthiness or fairness to a greater extent to attractive individuals compared to unattractive individuals (Dion et al., 1972; Ma, Xu, et al., 2015; Klebl et al., in press; Wilson \& Eckel, 2006). We found that this bias extends 
to judgments of moral violations; that is, people perceive unattractive individuals to be more likely to engage in moral violations than attractive individuals. Crucially, the present research extends past work by showing that attractiveness does not bias perceived likelihood to engage in moral violations uniformly but particularly biases moral character judgments pertaining to the moral domain of purity, relative to those unrelated to purity.

Our findings are consistent with previous work that has shown physical attractiveness evokes perceptions of purity (Klebl, Luo et al., 2021) and unattractiveness elicits the emotion of disgust (Klebl, Greenaway, et al., 2021; Kurzban \& Leary, 2001; Park et al., 2013; Ryan et al., 2012). Research on the human disease-avoidance system demonstrated that a diverse range of psychological mechanisms are involved in minimizing people's risk of getting infected by a contagious disease. First, aesthetic judgments such as judging an individual as attractive or unattractive are implicated in the disease-avoidance system, having the function to identify facial and bodily features that present heuristic cues for health or disease threat (Klebl, Greenaway, et al., 2021; Park et al., 2003; Rhodes, 2006). For example, people physically distance themselves from individuals with facial disfigurement and experience disgust towards them (Klebl, Greenaway, et al., 2021; Park et al., 2013; Rumsey et al., 1982; Ryan et al., 2012). Second, morality is implicated in the disease-avoidance system. People seek to minimize infection risk through moralizing behaviors that increase contamination threat (e.g., spitting on a sidewalk) or reduce contamination threat (e.g., purification rituals; Inbar \& Pizarro, 2014). The fact that the disease-avoidance system underpins both aesthetic judgments and moral judgments pertaining to the purity domain can explain why unattractive (vs. attractive) individuals are perceived as particularly more likely to engage in purity violations.

The magnitude of the effects found in present studies were within the range of the typical effect sizes found in similar studies on the Beauty-is-Good stereotype. The current 
investigation revealed small effect sizes for the bias of attractiveness on judgments of purity violations, whereas effect sizes found in previous studies investigating moral trait attributions to attractive (vs. unattractive) individuals ranged from very small to medium (Eagly et al., 1991; Feingold, 1992; Klebl et al., in press). However, we note that the current studies investigated perceived likelihood of individuals to engage in moral violations which limits direct comparisons with previous literature on trait attributions.

While the main focus of the present studies was to investigate whether people judge unattractive individuals to be more likely to engage in purity (vs. harm) violations than attractive individuals, we also examined the directionality of this effect. Previous research suggests that the Beauty-is-Good stereotype may be mostly due to a negative bias toward unattractive people rather than a positive bias toward attractive people (Griffin \& Langlois, 2006; Jaeger et al., 2018). Based on this research, our secondary prediction was that the effect may be due to individuals' unattractiveness. However, we found mixed support for this hypothesis. Study $1 \mathrm{~b}$ and Study 2 found that people judged attractive individuals to be less likely to engage in impure (vs. harmful) acts compared to both average-looking and unattractive individuals. In these studies, there was no significant difference between judgments of average-looking and unattractive individuals. However, Study 3, which used vignettes matched on moral wrongness, weirdness, and sociality, found-consistent with our secondary prediction - that unattractive individuals were judged to be more likely to engage in impure (vs. harmful) acts compared to both attractive and average-looking individuals. In this study there was no significant difference in judgments of attractive and average-looking individuals. This suggests that whereas people consistently judge unattractive individuals to be more likely to engage in impure (vs. harmful) acts than attractive individuals, judgments of average-looking individuals appear to be more context-dependent. Sometimes averagelooking individuals show effects similar to attractive individuals and sometimes they show 
effects similar to unattractive individuals. As we used different vignettes across studies, these differences in findings between Studies 2 and 3 may be due to idiosyncratic characteristics of the violations in question. In summary, while we found strong support for our main prediction that unattractive (vs. attractive) individuals would be judged as more likely to engage in purity violations than harm violations, we found inconsistent support for our secondary prediction that the effect would be driven by the lower end of the unattractiveness spectrum.

One limitation of the current investigation is that we compared purity violations to harm violations - that is, acts that inflict harm upon innocent persons. Harm intuitions have been argued to be of great importance in human morality and are distinct from the moral domain of purity (Graham et al., 2009). Thus, contrasting purity violations with harm violations presented a strong test for our hypothesis. Future research, however, should also compare purity violations to non-purity violations other than harm such as fairness violations (e.g., cheating in a card game) or authority violations (e.g., trying to undermine one's boss' ideas in front of others; see Clifford et al., 2015).

\section{Conclusion}

The current investigation sheds further light on the ways in which attractiveness biases person perception. We found that beyond biasing perceptions of moral character generally, physical attractiveness particularly biases moral character judgments in the purity domain. We proposed that this is because people implicitly associate attractiveness with purity and unattractiveness with impurity. Our findings contribute to a better understanding of the Beauty-is-Good stereotype and may help to better understand the psychological mechanisms involved in the stigmatization of unattractive people. 


\section{Open Practices Statement}

The data and materials of all studies are available at https://osf.io/m5g72/?view_only=d738c44e5de6487fa9d45c0ed07be5c8. We confirm that all measures, conditions, data exclusions and methods of determining sample sizes were reported. The pre-registration of Study $1 \mathrm{a}$ can be accessed at https://osf.io/qystm/?view_only=aa956910572840ffa392990d30b4f163, the pre-registration of Study $1 \mathrm{~b}$ can be accessed at https://osf.io/2sw86?view_only=76e7e150297e46e6a2456a0b9181aedc, the pre-registration of Study 2 is available at https://osf.io/8xygz?view_only=1cc6a6c2dd184c02a9a5a23490403998, and the preregistration of Study 3 is available at https://osf.io/3gbfs/?view_only=7792b32adbb945b89c1e53696d95e37a. 


\section{References}

Ambady, N., \& Rosenthal, R. (1992). Thin slices of expressive behavior as predictors of interpersonal consequences: A meta-analysis. Psychological Bulletin, 111(2), 256274. https://dx.doi.org/10.1037/0033-2909.111.2.256

Bascandziev, I., \& Harris, P. L. (2014). In beauty we trust: Children prefer information from more attractive informants. British Journal of Developmental Psychology, 32(1), 9499. https://doi.org/10.1111/bjdp.12022.

Bates, D., Mächler, M., Bolker, B., \& Walker, S. (2015). Fitting linear mixed-effects models using lme4. Journal of Statistical Software, 67(1), 1-48. https://dx.doi.org/10.18637/jss.v067.i01

Brambilla, M., Sacchi, S., Rusconi, P., Cherubini, P., \& Yzerbyt, V. Y. (2012). You want to give a good impression? Be honest! Moral traits dominate group impression formation. British Journal of Social Psychology, 51(1), 149-166.

Brambilla, M., Sacchi, S., Rusconi, P., Goodwin, G. (2021). The primacy of morality in impression development: Theory, research, and future directions. Advances in Experimental Social Psychology, 64. https://doi.org/10.1016/bs.aesp.2021.03.001

Carney, D. R., Colvin, C. R., \& Hall, J. A. (2007). A thin slice perspective on the accuracy of first impressions. Journal of Research in Personality, 41(5), 1054-1072. https://dx.doi.org/10.1016/j.jrp.2007.01.004

Chakroff, A., Dungan, J., \& Young, L. (2013). Harming ourselves and defiling others: What determines a moral domain? PloS One, 8(9), e74434. https://doi.org/10.1371/journal.pone.0074434

Chakroff, A., \& Young, L. (2015). Harmful situations, impure people: An attribution asymmetry across moral domains. Cognition, 136, 30-37. https://dx.doi.org/10.1016/j.cognition.2014.11.034 
Clifford, S., Iyengar, V., Cabeza, R., \& Sinnott-Armstrong, W. (2015). Moral foundations vignettes: A standardized stimulus database of scenarios based on moral foundations theory. Behavior Research Methods, 47(4), 1178-1198. https://doi.org/10.3758/s13428-014-0551-2

Dion, K., Berscheid, E., \& Walster, E. (1972). What is beautiful is good. Journal of Personality and Social Psychology, 24(3), 285-290. https://dx.doi.org/10.1037/h0033731

Eagly, A. H., Ashmore, R. D., Makhijani, M. G., \& Longo, L. C. (1991). What is beautiful is good, but...: A meta-analytic review of research on the physical attractiveness stereotype. Psychological Bulletin, 110(1), 109-128. https://dx.doi.org/10.1037/00332909.110.1.109.

Faul, F., Erdfelder, E., Buchner, A., \& Lang, A. G. (2009). Statistical power analyses using $\mathrm{G}^{*}$ Power 3.1: Tests for correlation and regression analyses. Behavior Research Methods, 41(4), 1149-1160. https://dx.doi.org/10.3758/BF03193146

Feingold, A. (1992). Good-looking people are not what we think. Psychological Bulletin, 111(2), 304-341. https://dx.doi.org/10.1037/0033-2909.111.2.304

Forgas, J. P., \& Laham, S. M. (2016). Halo effects. In R. F. Pohl (Ed.), Cognitive illusions: Intriguing phenomena in judgement, thinking and memory (pp. 276-290). Psychology Press.

Gerson, L. (Ed.). (2017). Plotinus: The Enneads (G. Boys-Stones, J. Dillon, R. King, A. Smith, \& J. Wilberding, Trans.). Cambridge University Press. doi:10.1017/9780511736490

Goodwin, G. P., Piazza, J., \& Rozin, P. (2014). Moral character predominates in person perception and evaluation. Journal of Personality and Social Psychology, 106(1), 148-168. https://dx.doi.org/10.1037/a0034726 
Graham, J., Haidt, J., \& Nosek, B. A. (2009). Liberals and conservatives rely on different sets of moral foundations. Journal of Personality and Social Psychology, 96(5), 10291046. https://dx.doi.org/10.1037/a0015141

Gray, K., \& Keeney, J. E. (2015). Impure or just weird? Scenario sampling bias raises questions about the foundation of morality. Social Psychological and Personality Science, 6(8), 859-868. https://doi.org/10.1177\%2F1948550615592241

Gray, K., Young, L., \& Waytz, A. (2012). Mind perception is the essence of morality. Psychological Inquiry, 23(2), 101-124. https://dx.doi.org/10.1080/1047840X.2012.651387

Griffin, A. M., \& Langlois, J. H. (2006). Stereotype directionality and attractiveness stereotyping: Is beauty good or is ugly bad? Social Cognition, 24(2), 187-206. https://dx.doi.org/10.1521/soco.2006.24.2.187

Gutierrez, R., \& Giner-Sorolla, R. (2007). Anger, disgust, and presumption of harm as reactions to taboo-breaking behaviors. Emotion, 7(4), 853-868. https://psycnet.apa.org/doi/10.1037/1528-3542.7.4.853

Haidt, J., Koller, S. H., \& Dias, M. G. (1993). Affect, culture, and morality, or is it wrong to eat your dog? Journal of Personality and Social Psychology, 65(4), 613-628. https://psycnet.apa.org/doi/10.1037/0022-3514.65.4.613

Heinzelmann, N. C., Weber, S. C., \& Tobler, P. N. (2020). Aesthetics and morality judgments share cortical neuroarchitecture. Cortex, 129, 484-495. https://dx.doi.org/10.1016/j.cortex.2020.04.018

Henderson, G. E. (2015). Ugliness: A cultural history. London: Reaktion Books. Horberg, E. J., Oveis, C., Keltner, D., \& Cohen, A. B. (2009). Disgust and the moralization of purity. Journal of Personality and Social Psychology, 97(6), 963-976. https://psycnet.apa.org/doi/10.1037/a0017423 
Hsee, C. K., Loewenstein, G. F., Blount, S., \& Bazerman, M. H. (1999). Preference reversals between joint and separate evaluations of options: A review and theoretical analysis. Psychological Bulletin, 125(5), 576-590. https://dx.doi.org/10.1037/00332909.125.5.576

Inbar, Y., \& Pizarro, D. A. (2014). Pollution and purity in moral and political judgment. In J. Wright \& H. Sarkissian (Eds.), Advances in experimental moral psychology (pp. 112129). Bloomsbury Academic.

Jaeger, B., Wagemans, F. M., Evans, A. M., \& Van Beest, I. (2018). Effects of facial skin smoothness and blemishes on trait impressions. Perception, 47(6), 608-625. https://doi.org/10.1177\%2F0301006618767258

Kant, I. (2000). Critique of the Power of Judgment (P. Guyer \& E. Matthews, Eds.).

Cambridge University Press. (Original work published in 1790).

Klebl, C., Greenaway, K. H., Rhee, J. J., \& Bastian, B. (2021). Ugliness judgments alert us to cues of pathogen presence. Social Psychological and Personality Science, 12(5), 617628. https://dx.doi.org/10.1177\%2F1948550620931655

Klebl, C., Luo, Y. \& Bastian, B. (2021). Beyond aesthetic judgment: Beauty increases moral standing via perceptions of purity. Personality and Social Psychology Bulletin. Advanced online publication.

Klebl, C., Rhee, J.J., Greenaway, K., Luo, Y., \& Bastian, B. (in press). Beauty goes down to the core: Attractiveness biases moral character attributions. Journal of Nonverbal Behavior.

Kurzban, R., \& Leary, M. R. (2001). Evolutionary origins of stigmatization: The functions of social exclusion. Psychological Bulletin, 127(2), 187-208. https://doi.org/10.1037//0033-2909.127.2.187 
Ma, D. S., Correll, J., \& Wittenbrink, B. (2015). The Chicago face database: A free stimulus set of faces and norming data. Behavior Research Methods, 47(4), 1122-1135. https://dx.doi.org/10.3758/s13428-014-0532-5

Ma, F., Xu, F., \& Luo, X. (2015). Children's and adults' judgments of facial trustworthiness: The relationship to facial attractiveness. Perceptual and Motor Skills, 121(1), 179198. https://dx.doi.org/10.2466\%2F27.22.PMS.121c10x1

Nisbett, R. E., \& Wilson, T. D. (1977). The halo effect: Evidence for unconscious alteration of judgments. Journal of Personality and Social Psychology, 35(4), 250-256. https://dx.doi.org/10.1037/0022-3514.35.4.250

Park, J. H., Faulkner, J., \& Schaller, M. (2003). Evolved disease-avoidance processes and contemporary anti-social behavior: Prejudicial attitudes and avoidance of people with physical disabilities. Journal of Nonverbal Behavior, 27(2), 65-87. https://doi.org/10.1177/1368430204046142

Park, J. H., Van Leeuwen, F., \& Chochorelou, Y. (2013). Disease-avoidance processes and stigmatization: Cues of substandard health arouse heightened discomfort with physical contact. The Journal of Social Psychology, 153(2), 212-228. https://doi.org/10.1080/00224545.2012.721812

Rhodes, G. (2006). The evolutionary psychology of facial beauty. Annual Review of Psychology, 57, 199-226. https://doi.org/10.1146/annurev.psych.57.102904.190208

Rozin, P. (1999). The process of moralization. Psychological Science, 10(3), 218-221. https://doi.org/10.1111\%2F1467-9280.00139

Rozin, P. (2000). Disgust. In M. Lewis \& J. M. Haviland-Jones (Eds.), Handbook of emotions (pp. 637-653). Guilford. 
Ryan, S., Oaten, M., Stevenson, R. J., \& Case, T. I. (2012). Facial disfigurement is treated like an infectious disease. Evolution and Human Behavior, 33(6), 639-646. https://doi.org/10.1016/j.evolhumbehav.2012.04.00

Schaller, M., \& Park, J. H. (2011). The behavioral immune system (and why it matters). Current Directions in Psychological Science, 20(2), 99-103. https://doi.org/10.1177\%2F0963721411402596

Tapp, C., Oaten, M., Stevenson, R. J., Occhipinti, S., \& Thandi, R. (2020). Is obesity treated like a contagious disease? Journal of Applied Social Psychology, 50(4), 205-212. https://doi.org/10.1111/jasp.12650

Thorndike, E. L. (1920). A constant error in psychological ratings. Journal of Applied Psychology, 4(1), 25-29.

Tsukiura, T., \& Cabeza, R. (2010). Shared brain activity for aesthetic and moral judgments: Implications for the Beauty-is-Good stereotype. Social Cognitive and Affective Neuroscience, 6(1), 138-148. http://dx.doi.org/10.1093/scan/nsq025

Tybur, J. M., Lieberman, D., \& Griskevicius, V. (2009). Microbes, mating, and morality: individual differences in three functional domains of disgust. Journal of Personality and Social Psychology, 97(1), 103-122. https://psycnet.apa.org/doi/10.1037/a0015474

Wheeler, L., \& Kim, Y. (1997). What is beautiful is culturally good: The physical attractiveness stereotype has different content in collectivistic cultures. Personality and Social Psychology Bulletin, 23(8), 795-800. https://doi.org/10.1177\%2F0146167297238001

Wilson, R. K., \& Eckel, C. C. (2006). Judging a book by its cover: Beauty and expectations in the trust game. Political Research Quarterly, 59(2), 189-202. https://dx.doi.org/10.1177\%2F106591290605900202 
Wolfe, N. D., Dunavan, C. P., \& Diamond, J. (2007). Origins of major human infectious diseases. Nature, 447(7142), 279-283. https://doi.org/10.1038/nature05775

Zebrowitz, L. A., \& Franklin Jr, R. G. (2014). The attractiveness halo effect and the babyface stereotype in older and younger adults: Similarities, own-age accentuation, and older adult positivity effects. Experimental Aging Research, 40(3), 375-393.

https://dx.doi.org/10.1080/0361073X.2014.897151 